but much more frequently in various complicated or associated, states. Now, I believe that $I$ have alone, of all other writers attempted to describe, or otherwise notice, most of these complications and associations.

When treating of the several topics connected with the pathology of paralysis, (ss. 166, et seg.), the views of Sir C. Bell, - Dr. M. Hall, and of others have been noticed by me as far as appeared requisite; not, however, confining myself to exclusive doctrines-to one-sided views-or to an obsolete physiology, but bestowing due regard on all matters which seemed of importance. Thus I have treated of-" The physical conditions of the brain and spinal chord"- "Of congestion of the venous sinuses seated between the theca of the chord and the bodies of the vertebra"-_." Of the various sympathetic phenomiena which occur in connection with paralysis"- and-" Of the mechanism and function of the spinal chord."

Under the latter heads I have given the minute anatomy of the spinal chord, and noticed the results of the researches of Foville, Dr. M. Hall, Dr. Todd, Mr. Grainger, Stilling, Van Deen, Budge, and Newport. As respects the physiology of the spinal chord, at least, I have adduced every thing deserving of notice up to the very recent communications of Mr. Newport in the latest volume of the Philosophical Transactions. You say, that my "physiological reminiscences are by no means up to the knowledge of the present day." Now assertions are easily made. I challenge you to adduce your proofs in support of them. It by no means follows that, because a practical writerold enough to know the fleeting character of nany doctrines, and the deceptive nature of painful and life-annihilating experiments on living dumb animals - chooses to estimate such doctrines and such experiments at something like a sober value, and to notice them accordingly, he should therefore be either ignorant of their existence, or disposed to exalt them above their sinking humility.*

('To be concluded in our next.)

\section{CASE OF FISTULA IN ANO.}

By T. N. MAGGIsox, Esq., M.D., M.R.C.S.Eng.

The following case of fistula in ano is drawn up at the earnest solicitation of a friend, who says that it may usefully exeite attention if published. I am sorry that I did not keep notes of the case at the time it was treated, not then thinking of its insertion in any public journal.

The patient, a young man, by occupation a clerk in a counting-house, applied to me on the 9th of March last, stating that he was very much afraid he was suffering from fistula. On examtnation, I found severe stricture of the rectum, and a fistula, into which the probe passed about an inch and a quarter, apparently blind. Owing to the severity of the stricture, I was unable to introduce more than the little finger into the rectum, and consequently could not with certainty ascertain the direction of the fistula. The patient would not hear of an operation, and begged $I$ would attempt anything rather than ltalk of it. I expressed my wilHngness to try what could be done, and, accordingly, on the 10th March, I commenced introducing rectum bougies. 'The first used was about the size of the little finger; it was introduced daily, and allowed to remain about a quarter of an hour; at the same time, a stick of lunar-caustic was daily applied to the fistula, being introduced as far as possible, and, on its withdrawal, a tent of lint was introduced, and allowed to remain.

The sphincter rapidly regained its normal extensibility, but a full-sized bougie was continued in daily use. I ought previously to have mentioned that on examination per anum, when effected, I found the probe pressing closely on the gut, about two inches within the rectum. The orifice of the fistula became gradually enlarged, so that, after the lapse of about six weeks, I had the satisfaction of seeing that the caustic had reached the end of the fistula, which by this time had diminished much in length, healthy granulations being seen at its extremity, and the point of the probe much less distinctly felt per anum. In a few days, by continuing the same treatment, the fistula rapidly disappeared; all that remained being a dense cord-like cicatrix in its former course.

The patient was not confined to bed, but continued his usual arocations.

Whickham, Durham, Oct. 10th, 1845.

* I am sorry that pressing engagements have prevented me from sending these remarks earlier.

Allow me to answer a question, by a correspondent, in one of the recent numbers of the THE LAscer, as to the subject "Percussion," by stating, that it is considered in the articles "Abdomen," and "Thorax."
TREATMENT OF HOUSE-MATD'S KNEE.

By Freberic R. Spackmax, Esq., M.B. Lond :, M.R.C.S., Fellow of the Royal Med. and Chir. Society.

In late Numbers of ThE LArcer some communications have appeared on the subject of "House-maid's knee." In the following case a modifeation of the puncturation treatment was adopted, and appears to possess some advantages over any $I$ have seen described :-

A servant-maid applied to me, in the beginning of June, having a bursa on one of her knees, fully as large as an orange. The integuments over it were tense, tender, and a good deal discoloured. A few days' rest, with the frequent application of cold water, removed all inflammatory action. On the I1th I introduced a grooved needle into the bursa, and, applying moderate pressure, removed a cor siderable portion of the liquid. A compress, the size of a half-crown, was firmly applied over the part by means of a bandage, and the whole direcied to be wetted, from time to time, with cold water, as the feelings of the patient prescribed. The bandage and comp.ess were re-applied daily, and the puncturation repeated on the 14 th, 16 th, and 26 th. No fluid escaped on the last-mentioned day; the tumour was then somewhat larger than a marble, and appeared to consist merely of hardened cellular t: ssue. 'The pressure was continued for some time, and the remains of the bursa slowly disappeared. 'There is now no trace of it whatever. The patient continued her usual work during the treatment, except on the days in which the needle was inserted; no inflammation supervened; the puncture had always healed perfectly on the second day; and, during the whole time, the pressure produced no discomfort. Harpenden, Oct. 23d, 1845 .

CASE OF SUSPENSION OF THE MENTAL FACULTIES, OF THE POWERS OF SPEECH, AND SPECLAC SENSES,

With the exception of sight and touch, occurring in a young woman, and continuing for many months, in consequence of her having accidentally fallen into a river, and been nearly drowned.

\section{By Robert DunN, Esq., Surgeon.}

MANT and curious are the instances which have been recorded of the arrest, suspension, and, at the time, apparent obliteration of some of the most ordinary functions of the nervous system, in consequence of a sudden shock, agitation, or fright.

Such cases are not more startling to the minds of common observers, than they are interesting to the physiologist and medical inquirer. The following plain narrative of an interesting case, which has come under my observation, may not be unworthy of the notice of my professional brethren. The subject $o_{i}^{n}$ the narrative is a young woman, now in the nineieenth year of her age, of a mixed temperament, the nervous with the lymphatic, and, as the medical attendant of the family, I have had frequent opportunities of seeing her since she was two years old. She was the first child of her parents and came prematurely into the world, about six weeks before the usual term of gestation. No reasonable hope was at first en uertained that she could live. She had not strength to suck, but the'milk, of which, fortunately, there was an ample supply, was drawn from the breasts, and she was fed with it by a spoon, and from a nursing bottle. At the end of six weeks, the completion of the reputed time of gestation, she was in convulsions for nearly an entire day, and all hope of her living was extinguished; but, nevertheless, she did survive, and from this time she began to thrive, to the great astonishment of every one who had seen her. She improved rapidly, and when, at four years old, she was inoculated for the small-pox she was a fine, strong, and healthy child, stouter and larger than most children at her age. A custom prevailed in the village to which she was removed, and where, indeed, she had been born, that whenever the small-pox broke out naturally, as it was termed, among the inhabitants, the other children of the village should be inoculated, if their parents or friends thought proper to have it done. At other times, inoculation was not permitted. She was, accordingly, inoculated, and had the small-pox so severely that it nearly cost her her life. She childhood, with the exception of scarlatina, which she had in a severe form when she was ten years old. After the attack of scarlatina, she enjoyed uniformly good health, up to the date of the present narrative, and grew up a strong, robust, and hearty young woman. Herperceptive powers were naturally quick,and, being largely endowed with the imitative faculty, she was put to 